\title{
Fontes de estresse, bem-estar psicológico e saúde entre estudantes de Odontologia: uma comparação entre fases pré-clínica e clínica e entre os sexos
}

\author{
Mariana Fernandes Muniz*; Francisco Wilker Mustafa Gomes Muniz**; Lidiany Karla Azevedo \\ Rodrigues***; Marcelo Bruno Lemos de Oliveira*; Isadora Daniel Barros*; Rosimary de Sousa \\ Carvalho****
}

* Cirurgiã(o)-dentista egressa(o) da Faculdade de Farmácia, Odontologia e Enfermagem, Universidade Federal do Ceará

** Doutor, Professor da Faculdade de Odontologia, Universidade Federal de Pelotas

*** Doutora, Professora da Faculdade de Farmácia, Odontologia e Enfermagem, Universidade Federal do Ceará

Recebido em 23/01/2019. Aprovado em 30/07/2019.

\begin{abstract}
RESUMO
O objetivo do estudo foi avaliar bem-estar psicológico, saúde geral e fontes de estresse de estudantes de Odontologia de quatro cursos, comparando as fases pré-clínica e clínica e os sexos. Três instrumentos foram aplicados: Dental Environment Stress (DES), Psychological General Well-Being Index (PGWB) e Health Survey (SF-36), respondidos por 203 estudantes, sendo 75 (37\%) em fase pré-clínica e 128 (63\%) em clínica. Desses, 59 (29\%) eram homens e 144 (71\%) mulheres. As comparações foram realizadas pelo teste de Mann-Whitney, adotando um nível de significância de 5\%. Nos domínios analisados pelo DES (performance acadêmica; dificuldades e inseguranças sobre o futuro profissional; responsabilidade com pacientes; fatores pessoais e institucionais; relações interpessoais), estudantes em fase pré-clínica demonstraram escores significativamente menores quando comparados a estudantes em fase clínica. No escore total de PGWB e em dois domínios (ansiedade e saúde geral), observou-se também escores menores em estudantes em fase pré-clínica. Quatro domínios do SF-36 (dor corporal, funcionamento físico, funcionamento social e papel físico), apresentaram significativa melhor saúde geral dos estudantes em fase pré-clínica. Entre os sexos, dois domínios do DES (performance acadêmica, dificuldade e insegurança sobre o futuro profissional) mostraram menores fatores estressores para o sexo masculino. O PGWB demonstrou diferença significativa em quatro domínios (estado deprimido; autocontrole; saúde geral; vitalidade), com melhor bem-estar psicológico para homens. O sexo masculino apresentou melhor vitalidade no domínio do SF-36. Concluiu-se que estudantes em fase pré-clínica e do sexo masculino demonstraram menores fontes de estresse, melhor bem-estar psicológico e percepção de saúde geral.

Descritores: Saúde Mental. Saúde. Estresse Ocupacional. Estudantes de Odontologia.
\end{abstract}




\section{INTRODUÇÃO}

Estudantes universitários estão expostos a diversos estímulos estressores inerentes ao ensino superior $^{1}$. A alta demanda educacional experimentada no ambiente acadêmico, aliada a fatores como falta de tempo para descanso, competitividade entre estudantes, afastamento do ambiente familiar e preocupações com o futuro mercado de trabalho exercem grande influência para o esgotamento emocional e físico de universitários ${ }^{2,3}$.

Dificuldade de memorização e concentração, diminuição do rendimento acadêmico, além de distúrbios psicológicos e físicos são sintomas comuns. Assim, o meio que deveria contribuir na edificação do conhecimento se torna, por vezes, limitador do desenvolvimento das habilidades pessoais e profissionais dos acadêmicos, incapacitando, seja física ou emocionalmente, a ampliação do seu potencial de aprendizado ${ }^{2,3}$.

Nesse contexto, a Odontologia tem sido apontada pela literatura como um dos campos de estudo mais desafiadores, exigentes e estressantes ${ }^{4}$. Estudos têm demonstrado resultados concordantes de que estudantes de Odontologia experimentam níveis significativamente mais altos de estresse que a população em geral ${ }^{5}$ e também em comparação a alunos de outras áreas da saúde, como a Medicina $^{6,7}$. Isso pode ser atribuído à natureza complexa e específica da educação odontológica, já que além da adaptação aos estímulos estressores, característicos do ambiente universitário, os estudantes de graduação em Odontologia precisam desenvolver habilidades manuais extremamente precisas, realizar atendimento clínico em pacientes ainda em treinamento ${ }^{8}$, ampliar suas competências interpessoais para o relacionamento com outros profissionais da área da saúde ${ }^{9}$, além do gerenciamento do tempo e do planejamento dos seus tratamentos ${ }^{10,11}$.

Pesquisadores de diversos países têm investigado os fatores de estresse específicos do treinamento odontológico e suas consequências psicológicas e físicas $5,6,10,12,13$. No entanto, no Brasil a temática tem sido pouco abordada. Além disso, pouco se conhece, em âmbito nacional e internacional, sobre possíveis diferenças referidas na intensidade de estresse entre estudantes de diferentes fases do curso de Odontologia (préclínica e clínica) e entre os sexos. Considerando que as características divergentes entre esses grupos de estudantes podem influenciar a percepção do estresse, bem como seu impacto no bem-estar e na saúde geral, é fundamental que se compreenda quais grupos estão mais vulneráveis e que se discuta estratégias de enfrentamento e gerenciamento desses fatores estresseores, de forma a permitir a melhoria da qualidade geral de ensino e de aprendizado no sistema de educação odontológica.

O objetivo do presente estudo foi comparar as fontes de estresse, o bem-estar psicológico e a saúde geral entre alunos do curso de Odontologia das fases pré-clínica e clínica e entre os sexos, de quatro instituições de ensino superior do estado do Ceará, Brasil.

\section{METODOLOGIA}

O estudo obedeceu aos critérios exigidos pela resolução 466/2002 do Conselho Nacional de Saúde $^{14}$. As identidades dos participantes foram mantidas em sigilo e as informações confidencialmente garantidas. $\mathrm{O}$ protocolo do estudo foi aprovado pelo Comitê de Ética da Universidade Federal do Ceará sob o $\mathrm{n}^{\circ}$ $953.335 / 2015$. Todos os voluntários assinaram um termo de consentimento livre e esclarecido.

Trata-se de estudo transversal, de natureza quantitativa. Participaram do estudo acadêmicos de Odontologia de quatro instituições de ensino superior do Ceará: Universidade de Fortaleza e Universidade Federal do Ceará - campus Fortaleza, ambas com sede na capital do estado (Fortaleza); Centro Universitário Católica de 
Quixadá e Universidade Federal do Ceará campus Sobral, ambos com sede no interior do estado. Para serem incluídos, os participantes precisariam ter no mínimo 18 anos de idade e estarem matriculados regularmente no ano de 2015. Além disso, eles precisaram preencher as seguintes variáveis do questionário: sexo, fase acadêmica e instituição na qual estava matriculado.

Os estudantes que responderam a todas essas variáveis, porém não responderam aos três instrumentos utilizados no questionário (Dental Environment Stress, Psycological General Well Being e Health Survey) foram excluídos da análise de dados.

Todas as instituições envolvidas no presente estudo possuem um grupo fechado na rede social Facebook e possuem um e-mail gerenciado apenas pelos estudantes de cada turma. De fevereiro a maio de 2015 foram enviados convites aos estudantes por meio dessas mídias sociais e emails, explicando os principais objetivos e os critérios de inclusão do estudo. Os voluntários foram convidados a responder enviando seu e-mail pessoal para um dos pesquisadores envolvidos no presente estudo. Poderia também marcar um encontro presencial para responder ao questionário em formato impresso. A confirmação da matrícula do aluno no ano de 2015 foi feita por contato com as coordenações dos cursos acima citados.

Como todos os estudantes de Odontologia (1500 alunos regularmente matriculados, no ano de 2015) foram convidados a participar, um cálculo de tamanho de amostra não foi realizado previamente à realização do estudo.

Foi determinado, a posteriori, um poder de 99,96\% e 78,97\% para as comparações entre as fases acadêmicas e os sexos, respectivamente. Para tanto, em cada análise, utilizou-se a média e desvio padrão dos escores encontrados no instrumento Dental Environment Stress (DES) em um intervalo de confiança de $95 \%$. Para alcançar $80 \%$ de poder na análise entre os sexos, seriam necessários 207 estudantes de Odontologia, o que está próximo do tamanho final da amostra de 203 participantes do presente estudo.

$\mathrm{O}$ instrumento DES tem sido amplamente utilizado, sendo considerado o melhor para avaliar e quantificar os estressores específicos do ambiente odontológico $^{14}$. Seu formato validado e traduzido para a população brasileira foi aplicado no presente estudo $^{15}$. Essa ferramenta é constituída por 36 itens divididos em cinco domínios, com respostas baseadas em escala Likert de quatro pontos, sendo 1 - não estressante e 4 - muito estressante. Os cinco domínios são: "performance acadêmica", "dificuldades e inseguranças sobre o futuro profissional", "responsabilidade com os pacientes", "fatores pessoais e institucionais" e "relações interpessoais".

O índice Psychological General Well-Being (PGWB), em seu formato traduzido e validado para uma população portuguesa ${ }^{16,17}$ foi aplicado nesse estudo para medir o bem-estar psicológico e o sofrimento geral nos estudantes de Odontologia. É composto por 22 perguntas incluídas em seis domínios. A pontuação original por item varia de 0 a 5, sendo zero para uma experiência mais negativa e cinco pontos para uma experiência mais positiva, com uma pontuação máxima total de até 110 pontos. O PGWB é composto por seis domínios: "ansiedade", "humor deprimido", "bem-estar positivo", "autocontrole", "saúde geral" e "vitalidade".

O formato traduzido e validado para uma amostra brasileira do instrumento Health Survey (SF-36) foi utilizado para avaliação da saúde geral dos participantes ${ }^{18}$. Essa ferramenta é constituída por 36 itens agrupados em oito domínios. Cada item apresenta um escore final de 0 a 100, no qual zero corresponde ao pior estado geral de saúde e 100 o melhor estado geral de saúde. O SF-36 compreende os seguintes domínios: função física, papel físico, dor corporal, saúde geral, vitalidade, função social, papel emocional e saúde mental. 
Esse estudo comparou os domínios dos três instrumentos entre os estudantes de fase pré-clínica e clínica e entre os sexos. A normalidade da distribuição dos dados foi testado pelo teste de Shapiro-Wilk. Como uma distribuição não normal foi identificada, o teste de Mann-Whitney foi aplicado em todas as comparações. Para as variáveis categóricas, o teste de qui-quadrado foi utilizado. A confiabilidade dos três instrumentos foi previamente avaliada pelo coeficiente alfa de Cronbach ${ }^{19}$.

A análise estatística foi realizada utilizando o SPSS versão 21 para Windows (IBM® SPSS Statistics, Nova York, EUA), adotando-se um nível de significância de 5\% para todas as análises.

\section{RESULTADOS}

No total, 237 e-mails foram recebidos e o questionário foi enviado para apenas 231 alunos, pois, seis desses alunos não estavam regularmente matriculados no ano de 2015. Duzentos e três alunos retornaram o questionário (taxa de resposta de 87,88\%). Destes, 98 (48,28\%) e $105(51,72 \%)$ questionários foram, respectivamente, preenchidos em formato impresso e eletrônico. Dos 203 estudantes que responderam ao questionário, 75 (37\%) cursavam a fase pré-clínica e 128 (63\%) a fase de clínica. Destes, 59 (29\%) eram do sexo masculino e $144(71 \%)$ do sexo feminino. As tabelas 1 e 2 descrevem as características demográficas da amostra segundo a fase acadêmica e o sexo, respectivamente.

Nas comparações entre os sexos, não se evidenciou diferenças estatisticamente significativas para as variáveis idade, curso e fase acadêmica dos participantes. Também não foram encontradas diferenças estatisticamente significativas entre as fases acadêmicas.

Tabela 1. Características demográficas da amostra segundo a fase do curso

\begin{tabular}{|c|c|c|c|c|}
\hline & & $\begin{array}{l}\text { Estudantes em } \\
\text { fase pré-clínica } \\
(n=75)\end{array}$ & $\begin{array}{l}\text { Estudantes em } \\
\text { fase clínica } \\
(n=128)\end{array}$ & Valor-p \\
\hline Idade (anos) & $\begin{array}{l}\text { Média } \pm \mathrm{DP} \\
\text { (média-min.;max.) }\end{array}$ & $\begin{array}{l}22,00 \pm 3,16 \\
(22-18 ; 32)\end{array}$ & $\begin{array}{l}22,23 \pm 5,44 \\
(21-18 ; 50)\end{array}$ & $0,739^{\#}$ \\
\hline Sexo & $\begin{array}{l}\text { Masculino - n }(\%) \\
\text { Feminino - } \mathrm{n}(\%)\end{array}$ & $\begin{array}{l}22(29,3) \\
53(70,7)\end{array}$ & $\begin{array}{l}37(28,9) \\
91(71,1)\end{array}$ & $0,948 *$ \\
\hline Tipo de escola & $\begin{array}{l}\text { Pública }-\mathrm{n}(\%) \\
\text { Privada }-\mathrm{n}(\%)\end{array}$ & $\begin{array}{l}36(48,0) \\
39(52,0)\end{array}$ & $\begin{array}{l}56(43,8) \\
72(56,3)\end{array}$ & $0,557 *$ \\
\hline
\end{tabular}

*teste qui-quadrado; " teste t para amostras independents

Tabela 2. Características demográficas da amostra segundo sexo

\begin{tabular}{lllll}
\hline & & Sexo Masculino & Sexo Feminino & Valor-p \\
\hline Idade (anos) & Média \pm DP & $22,19 \pm 5,06$ & $22,13 \pm 4,58$ & $0,933^{\#}$ \\
& (média-min.;max.) & $(21,5-18 ; 50)$ & $(21-18 ; 50)$ & \\
Fase acadêmica & Fase Pre-clínica-n $(\%)$ & $22(37,3)$ & $53(36,8)$ & $0,948^{*}$ \\
& Fase Clínica - n (\%) & $37(62,7)$ & $91(63,2)$ & \\
Tipo de escola & Pública - n (\%) & $30(50,8)$ & $62(43,1)$ & $0,311^{*}$ \\
& Privada - n $(\%)$ & $29(49,2)$ & $82(56,9)$ & \\
\hline
\end{tabular}

*teste qui-quadrado; "teste t para amostras independentes. 


\section{Instrumento DES}

A tabela 3 apresenta os escores do instrumento DES de acordo com seus domínios na comparação entre as fases acadêmicas e os sexos. Todos os domínios apresentaram diferenças estatisticamente significativas entre os grupos. Os escores totais médios apresentaram também diferença estatisticamente significativa, com menor pontuação para os estudantes em fase pré-clínica $(76,20 \pm 15,65)$, em comparação ao dos estudantes em fase clínica $(89,48 \pm 19,52)$.

Com relação às comparações entre os sexos, apenas os domínios "performance acadêmica" e "dificuldades e insegurança sobre o futuro profissional" apontaram diferenças significativas, com o sexo feminino apresentando em ambos domínios os maiores escores. O escore total, quanto aos sexos, foi de $79,15( \pm 17,07)$ para homens, e $86,79( \pm 19,70)$ para mulheres, sendo essa diferença estatisticamente significativa $(\mathrm{p}=0.006)$.

Tabela 3. Escores obtidos de acordo com as fases acadêmicas e sexos por meio do instrumento DES

\begin{tabular}{|c|c|c|c|c|}
\hline DES - domínios & Fase pré-clínica & Fase clínica & Sexo masculino & Sexo feminino \\
\hline Performance acadêmica & $26,79(5,79)^{*}$ & $28,95(6,21)^{*}$ & $26,75(5,30)^{*}$ & $28,72(6,37)^{*}$ \\
\hline $\begin{array}{l}\text { Dificuldades e } \\
\text { inseguranças sobre o } \\
\text { futuro profissional }\end{array}$ & $13,53(4,12)^{*}$ & $15,40(5,26)^{*}$ & $12,86(4,64)^{*}$ & $15,47(4,87)^{*}$ \\
\hline $\begin{array}{l}\text { Responsabilidade com } \\
\text { os pacientes }\end{array}$ & $5,36(2,16)^{*}$ & $9,81(2,73)^{*}$ & $7,86(3,17)$ & $8,28(3,38)$ \\
\hline $\begin{array}{l}\text { Fatores pessoais e } \\
\text { institucionais }\end{array}$ & $16,87(4,28)^{*}$ & $20,30(5,61)^{*}$ & $17,85(4,67)$ & $19,52(5,69)$ \\
\hline Relações interpessoais & $13,65(4,58)^{*}$ & $15,02(4,67)^{*}$ & $13,83(5,00)$ & $14,80(4,51)$ \\
\hline Escore total & $76,20(15,65)^{*}$ & $89,48(19,52)^{*}$ & $79,15(17,07)^{*}$ & $86,79(19,70)^{*}$ \\
\hline
\end{tabular}

\section{Instrumento PGWB}

Em relação às comparações entre fases acadêmicas, apenas dois domínios ("ansiedade" e "saúde geral") apresentaram diferenças significativas entre os grupos, sendo os maiores escores encontrados nos estudantes da fase préclinica quando comparados aos da fase clínica no instrumento PGWB. As diferenças dos escores totais médios entre os estudantes da fase pré-clínica $(63,24 \pm 12,85)$ e da fase clínica $(59,09 \pm 14,65)$ demonstraram ser estatisticamente significativas $(\mathrm{p}=0.015)$ (tabela 4).

A comparação entre os sexos no instrumento PGWB demonstrou diferenças significativas em quatro domínios (humor deprimido; autocontrole; saúde geral; vitalidade). Em todos os domínios, o sexo feminino apresentou escores significativamente menores, demonstrando maior impacto no bemestar psicológico. Os escores totais médios para sexo masculino foram $(65,36 \pm 15,45)$ e para o sexo feminino $(58,68 \pm 13,10)$, que também se demonstraram significativamente diferentes $(\mathrm{p}=0,002)$ (tabela 4).

\section{Instrumento SF-36}

Em relação ao SF-36, foram constatadas diferenças significativas em 4 domínios relacionados às fases acadêmicas. Novamente, alunos em fase pré-clínica apresentaram 
significativamente menores escores em relação aos domínios "função física", "papel comparação aos que estavam em fase clínica, em físico", "dor corporal" e "função social" (tabela 5).

Tabela 4. Escores obtidos de acordo com as fases acadêmicas e sexos por meio do instrumento PGWB

\begin{tabular}{lllll}
\hline PGWB - domínios & Fase pré-clínica & Fase clínica & Sexo masculino & Sexo feminino \\
Ansiedade & $13,01(3,79)^{*}$ & $11,63(4,91)^{*}$ & $13,08(5,10)$ & $11,76(4,29)$ \\
Estado deprimido & $10,15(2,94)$ & $9,49(3,12)$ & $10,70(3,16)^{*}$ & $9,34(2,95)^{*}$ \\
Bem-estar positivo & $10,63(2,72)$ & $10,87(3,39)$ & $11,25(3,14)$ & $10,58(3,14)$ \\
Auto controle & $9,33(2,46)$ & $9,55(2,77)$ & $10,12(2,63)^{*}$ & $9,21(2,64)^{*}$ \\
Saúde geral & $9,72(1,97)^{*}$ & $8,04(2,78)^{*}$ & $9,38(2,50)^{*}$ & $8,37(2,65)^{*}$ \\
Vitalidade & $10,40(2,63)$ & $9,50(3,11)$ & $10,83(3,35)^{*}$ & $9,42(2,70)^{*}$ \\
Escore total & $63,24(12,85)^{*}$ & $59,09(14,65)^{*}$ & $65,36(15,45)^{*}$ & $58,68(13,10)^{*}$ \\
\hline *p<0,05 para as comparações entre as fases acadêmicas ou sexos. Os valores estão expressos em média (desvio \\
padrão).
\end{tabular}

Tabela 5. Escores obtidos de acordo com as fases acadêmicas e sexos por meio do instrument SF-36

\begin{tabular}{lllll}
\hline SF-36 - domínios & Fase pré-clínica & Fase clínica & Sexo masculino & Sexo feminino \\
\hline Função física & $78,80(17,47)^{*}$ & $72,22(21,52)^{*}$ & $75,85(20,37)$ & $74,17(20,36)$ \\
Papel físico & $49,00(29,48)^{*}$ & $39,96(35,53)^{*}$ & $44,83(34,34)$ & $42,71(33,43)$ \\
Dor corporal & $70,65(19,67)^{*}$ & $63,09(22,18)^{*}$ & $67,59(21,27)$ & $65,22(21,70)$ \\
Saúde geral & $62,99(17,56)$ & $60,28(18,47)$ & $64,66(17,62)$ & $59,93(18,23)$ \\
Vitalidade & $47,67(16,99)$ & $45,98(18,43)$ & $51,81(18,89)^{*}$ & $44,51(17,09)^{*}$ \\
Função social & $64,83(20,42)^{*}$ & $58,76(21,78)^{*}$ & $63,36(20,53)$ & $60,07(21,79)$ \\
Papel emocional & $52,89(33,82)$ & $50,92(40,02)$ & $53,45(38,46)$ & $50,93(37,59)$ \\
Saúde mental & $60,64(14,89)$ & $58,90(18,53)$ & $62,48(18,06)$ & $58,36(16,84)$ \\
Escore total & $487,47(92,46)$ & $449,91(120,28)$ & $483,60(121,90)$ & $455,90(107,20)$ \\
\hline * p<0,05 para as comparações entre as fases acadêmicas ou sexos. Os valores estão expressos em média (desvio \\
padrão).
\end{tabular}

Em relação aos sexos, apenas o domínio "vitalidade" apresentou diferença significativa entre os grupos. Os estudantes do sexo masculino apresentaram maior pontuação, com escore médio de 51,81( $\pm 18,89)$, já as estudantes do sexo feminino apresentaram um escore médio de $44,51( \pm 17,09)$. Não houve diferença significativa para o escore total médio desse instrumento, seja nas comparações entre fase acadêmica $(\mathrm{p}=0.055)$ ou entre sexos $(\mathrm{p}=0.065)$ (tabela 5$)$.

\section{DISCUSSÃO}

O presente estudo objetivou verificar fontes de estresse, bem-estar psicológico e aspectos de saúde geral em estudantes de Odontologia de quatro Universidades do estado do Ceará. Foi verificado que estudantes da fase clínica e do sexo feminino apresentam significativo impacto nas fontes de estresse, no bem-estar psicológico e em alguns aspectos da saúde geral quando comparados com estudantes da fase pré-clínica e do sexo masculino, respectivamente.

Estudantes de Odontologia experimentam níveis consideráveis de estresse durante seu treinamento, o que pode ter implicações em seu bem-estar profissional, emocional, físico, social e futuro $^{20}$. Contudo, a literatura carece de informações que identifiquem qual fase do curso e 
qual sexo estão expostos a esses estressores de maneira mais crítica, o que implicaria em um maior impacto em seu bem-estar psicológico e na saúde geral. Esse estudo é um dos primeiros a comparar estressores entre as fases acadêmicas e entre os sexos em estudantes brasileiros.

No que se refere às comparações entre as fases acadêmicas, os cinco domínios do instrumento DES apresentaram diferenças estatisticamente significativas, além do escore total médio, com maior pontuação para estudantes em fase clínica, representando percepção de fontes estressoras mais intensas para esse grupo de acadêmicos.

Esta investigação corrobora outras encontradas na literatura. Foi reportado que quanto mais tempo os alunos estão inseridos no curso de Odontologia, mais acentuados são os fatores estressores referidos. Além disso, o aumento nos níveis de estresse desses estudantes pode refletir um efeito cumulativo ou, alternativamente, sugerir que cada ano progressivo de treinamento se torna mais difícil e estressante ${ }^{21}$. Outra pesquisa realizada em cinco cursos de Odontologia da Europa demonstrou que estudantes no primeiro ano percebiam significativamente menor intensidade de estresse em comparação aos do último $a^{22}$. Estudos realizados em outros países, como os Estados Unidos, Japão, Argentina e Turquia, comprovaram níveis de estresse mais intensos em estudantes de fase clínica ${ }^{23-26}$. O presente estudo está de acordo com esses resultados, já que demonstrou maior intensidade de estresse percebido em estudantes da fase clínica.

Não obstante, outras pesquisas demonstraram que os estudantes que estão no momento de transição entre fase pré-clínica e clínica apresentaram maiores escores relacionados a fatores estressores específicos ${ }^{4,21,27,28}$. A maior exigência envolvendo o manejo do paciente pode ser outra explicação para as maiores fontes de estresses percebidas pelos estudantes. Esse grupo precisa ser capaz de lembrar o conhecimento teórico adquirido na fase pré-clínica, colocá-lo em prática em pacientes pela primeira vez, devem ser responsáveis por seus cuidados e realizar tratamentos dentários irreversíveis ${ }^{3}$. O domínio "Responsabilidade com os pacientes", no presente estudo, apresentou diferença significativa entre os acadêmicos das diferentes fases do curso, indicando que o dever do aluno relacionado aos cuidados com o paciente é uma das causas de maior nível de estresse percebido entre o grupo de estudantes em fase clínica.

Os dados obtidos nesse estudo, a partir instrumentos PGWB e SF-36, utilizados para análise do bem-estar psicológico e de saúde geral, respectivamente, indicaram uma situação desfavorável para os estudantes em fase clínica. $\mathrm{O}$ instrumento PGWB apontou valores significativos nos domínios "ansiedade" e "saúde geral", e o instrumento SF-36, nos domínios "função física", "papel físico", "dor corporal" e "função social". Em ambas as ferramentas, foram encontradas maiores pontuações para alunos em fase préclínica, o que significa uma melhor percepção do bem-estar psicológico e da saúde geral. $\mathrm{O}$ aumento da complexidade do curso de Odontologia a partir da fase clínica inclui exigências físicas ${ }^{26}$. $\mathrm{Na}$ área odontológica, muitos procedimentos exigem movimentos corporais de torção e estática por longos períodos de tempo ${ }^{27}$. Alunos em fase clínica estão mais expostos a dificuldades físicas geradas pela atividade clínica.

Na comparação entre os sexos, os domínios "performance acadêmica" e "dificuldades e insegurança sobre o futuro profissional" do instrumento DES demonstraram valores significativamente maiores para as mulheres, indicando maior percepção de fatores estressores quando comparados com estudantes do sexo masculino. Esse resultado está de acordo com estudos anteriores que indicam a influência do sexo na percepção dos fatores estressores, estando às 
mulheres em uma situação mais vulnerável ${ }^{11,29-31}$. Relata-se que a construção social da masculinidade influencia os homens a serem menos propensos a relatar o estresse ${ }^{31}$. Contudo, outras variáveis históricas, culturais e biológicas devem ser consideradas.

A reflexão de que estudantes brasileiras de Odontologia estão inseridas nessa realidade social deve ser considerada para a análise dos resultados obtidos no presente estudo. $\mathrm{O}$ acúmulo de tarefas para o sexo feminino pode influenciar no estresse percebido relacionado ao desempenho acadêmico e às inseguranças sobre o mercado de trabalho, apontado nos dados do instrumento DES. Outros estudos devem investigar melhor essa interferência social no estresse percebido em mulheres inseridas no treinamento odontológico.

Constatou-se, também, que os domínios "humor deprimido", "autocontrole", "saúde geral" e "vitalidade", além do escore total médio, do instrumento PGWB, e o domínio "vitalidade" do instrumento SF-36 demonstraram diferenças significativas nas comparações entre sexos, com escores mais altos para o sexo masculino, o que indica um melhor panorama de bem-estar psicológico e de percepção da saúde geral para os homens. As diferenças entre os sexos podem estar relacionadas às flutuações cíclicas de estrogênio e progesterona e favorecem respostas ao estresse, conferindo às mulheres suscetibilidade para ansiedade e depressão ${ }^{32}$. As pontuações significativas dos domínios citados neste estudo podem estar relacionadas a essa singularidade feminina.

A influência da questão hormonal das mulheres é uma característica natural da própria fisiologia do corpo feminino, que pode repercutir na sua forma de lidar com o estresse, não devendo ser ignorada. A construção histórica da Odontologia no Brasil foi predominantemente masculina ${ }^{33}$, o que pode naturalizar uma negação à compreensão das peculiaridades femininas.
Discussões dentro das instituições de ensino superior sobre a temática podem ser espaços de compreensão e mudança dessa realidade.

Além dessas atitudes, diversas outras estratégias podem ser adotadas no intuito de diminuir os fatores estressores desses estudantes, como exercícios físicos ${ }^{34}$, terapia cognitiva baseada em mindfulness ${ }^{35}$ ou com o uso de musicoterapia $^{36}$. A literatura ainda aponta que a espiritualidade pode desempenhar um importante papel nos melhores estados de bem-estar entre estudantes de Odontologia ${ }^{37}$. Esses resultados podem ser utilizados em implementações de estratégias na intenção de contribuir com o enfretamento de fatores estressores dos estudantes do Curso de Odontologia. No entanto, deve ser colocado em perspectiva que alguns desses fatores não estão relacionados a fontes acadêmicas, pois eliminar todos os estressores é uma tarefa complexa.

Apesar dos achados relevantes, o presente estudo apresenta algumas limitações. $\mathrm{O}$ seu desenho transversal, o qual não permite temporalidade, deve ser considerado no momento da interpretação dos dados. Além disso, uma alta validade externa pode não ser identificada, pois a estratégia de recrutamento para essa pesquisa contou apenas com envio de e-mails e contatos em redes sociais. Apesar disso, os dois cálculos de poder, para as duas comparações efetuadas, demonstraram um poder estimado de aproximadamente $80 \%$ ou superior. Considerouse, portanto, que o número de estudantes incluídos foi adequado.

\section{CONCLUSÕES}

Estudantes em fase pré-clínica e do sexo masculino apresentaram menor percepção de estresse, melhor bem-estar psicológico e melhor percepção de sua saúde geral quando comparados aos da fase clínica e do sexo feminino, respectivamente. Entretanto, mais 
estudos são necessários para melhor avaliação e compreensão das consequências dos fatores estressores em estudantes de Odontologia, contribuindo para melhorias no sistema de educação odontológica.

\section{AGRADECIMENTOS}

Esse estudo foi parcialmente suportado pela Coordenação de Aperfeiçoamento de Pessoal de Nível Superior (CAPES) por meio da utilização do Portal Periódicos CAPES. O autor Marcelo B. L. Oliveira foi bolsista de iniciação científica PIBIC da Universidade Federal do Ceará durante o desenvolvimento do estudo. Agradecemos aos coordenadores dos cursos à época da pesquisa: Fabricio Bitu (UFC - Fortaleza), Mariana Farias (UFC - Sobral), Fernando André (UNIFOR), Lívia Barros (Unicatólica) e Iracema Matos (UFC Sobral).

\footnotetext{
ABSTRACT

Sources of stress, psychological well-being and health among dental students: a comparison between preclinical and clinical phases and between sexes

The aim of this study was to evaluate psychological well-being, overall health, and sources of stress among dental students from four Doctor of Dental Surgery (DDS) programs, comparing the preclinical and clinical phases and sexes. Three instruments were applied: Dental Environment Stress (DES), Psychological General Well-Being Index (PGWB) and Health Survey (SF-36), answered by 203 students, with $75(37 \%)$ in the preclinical phase and $128(63 \%)$ in the clinical one. Among them, 59 were male (29\%) and 144 were female (71\%). Comparisons were performed using the Mann-Whitney test, adopting a significance level of $5 \%$. In the domains analyzed using DES (academic performance, difficulties and insecurities about their professional future, responsibilities with patients, personal and institutional factors, interpersonal relationships), preclinical students showed significantly lower scores than students
}

enrolled in the clinical phases. For PGWB total score and in two domains (anxiety and general health), lower scores were also observed in preclinical students. Four domains of SF-36 (bodily pain, physical functioning, social functioning and role-physical) showed significantly better overall health for preclinical students. Among the sexes, two DES domains (academic performance and difficulties and insecurities about their professional future) showed lower stressors for males. PGWB showed a significant difference in four domains (depressed mood, self-control, general health and vitality), with better psychological well-being for males. Males showed better vitality in the SF-36 domain. Concluding, preclinical and male students showed lower sources of stress, better psychological well-being and better perception of their overall health.

Descriptors: Mental Health. Health. Occupational Stress. Dentistry students.

\section{REFERÊNCIAS}

1. Tosevski DL, Milovancevic MP, Gajic SD. Personality and psychopathology of university students. Curr Opin Psychiatry. 2010; 23(1):48-52.

2. de Souza Monteiro CF, de Medeiros Freitas JF, Ribeiro AAP. Estresse no cotidiano acadêmico: o olhar dos alunos de enfermagem da Universidade Federal do Piauí. Esc Anna Nery Rev Enferm. 2007; 11(1):66-72.

3. Elani HW, Allison PJ, Kumar RA, Mancini L, Lambrou A, Bedos C. A systematic review of stress in dental students. J Dent Educ. 2014; 78(2):226-42.

4. Polychronopoulou A, Divaris K. Dental students' perceived sources of stress: a multi-country study. J Dent Educ. 2009; 73(5):631-9.

5. Cooper CL, Watts J, Kelly M. Job satisfaction, mental health, and job stressors among general dental practitioners in the UK. Br Dent J. 1987;162(2):77-81. 
6. Birks Y, McKendree J, Watt I. Emotional intelligence and perceived stress in healthcare students: a multi-institutional, multi-professional survey. BMC Med Educ. 2009; 9(1):61. Doi: 10.1186/1472-6920-961.

7. Murphy RJ, Gray SA, Sterling G, Reeves K, DuCette J. A comparative study of professional student stress. J Dent Educ. 2009; 73(3):328-37.

8. Alzahem AM, Van der Molen HT, Alaujan AH, De Boer BJ. Stress management in dental students: a systematic review. Adv Med Educ Pract. 2014; 5:167. Doi: 10.2147/AMEP.S46211.

9. Muirhead V, Locker D. Canadian dental students' perceptions of stress and social support. Eur J Dent Educ. 2008; 12(3):1448.

10. Divaris K, Barlow PJ, Chendea SA, Cheong WS, Dounis A, Dragan IF, Mo'nes M. The academic environment: the students' perspective. Eur J Dent Educ. 2008; 12 (Suppl 1):120-30.

11. Fonseca J, Divaris K, Villalba S, Pizarro S, Fernandez M, Codjambassis A, Polychronopoulou A. Perceived sources of stress amongst $\mathrm{C}$ hilean and $\mathrm{A}$ rgentinean dental students. Eur J Dent Educ. 2013; 17(1):30-8.

12. Alzahem AM, Van der Molen HT, Alaujan AH, Schmidt HG, Zamakhshary MH. Stress amongst dental students: a systematic review. Eur J Dent Educ. 2011; 15(1):8-18.

13. Dahan H, Bedos C. A typology of dental students according to their experience of stress: a qualitative study. J Dent Educ. 2010; 74(2):95-103.

14. Brasil. Agência Nacional de Saúde. Resolução $n^{\circ}$. 466, de 12 de dezembro de 2012. Diretrizes e normas regulamentadoras de pesquisas envolvendo. [Acesso em 23 de janeiro de 2019]. Disponível em: http://bvsms.saude.gov.br/bvs/saudelegis/cn s/2013/res0466_12_12_2012.html.

15. Sangiorgio JPM, Araujo PM, Navarro $\mathrm{CH}$, Zen IR, da Costa SC, Ribeiro PHV, DezanGarbelini, CC. Dental environment stress: Findings among Lusophone dental students. Pesq Bras Odontoped Clin Integr. 2016 16(1):411-24.

16. Chassany O, Dimenäs E, Dubois D, Wu A, Dupuy H. The psychological general wellbeing index (pgwbi) user manual. Lyon, France: Mapi Research Institute; 2004.

17. Espírito Santo RPDSD. Bem-estar Psicológico: Validação da Escala Psychological General Well-being para a população portuguesa. Porto. Dissertação [Mestrado em Psicologia Clínica e da Saúde] - Universidade Lusófona do Porto; 2015.

18. Ciconelli RM, Ferraz MB, Santos W, Meinão I, Quaresma MR. Tradução para o português e validação do questionário genérico de avaliação de qualidade de vida Medical Outcomes Study 36-Item ShortForm Health Survey (SF-36). Rev Bras Reumatol. 1999; 39(3):143-50.

19. Muniz FWMG, Oliveira MBL, Barros ID, Oliveira PMC, Rodrigues LKA, Carvalho RS. Stressors, psychological well-being, and overall health amongst students from public and private dental schools. Braz J Oral Sci. 2018; 17: e181210. Doi: 10.20396/ bjos.v17i0.8654216.

20. Polychronopoulou A, Divaris K. A longitudinal study of Greek dental students' perceived sources of stress. J Dent Educ. 2010; 74(5):524-30.

21. Naidu RS, Adams JS, Simeon D, Persad S. Sources of stress and psychological disturbance among dental students in the West Indies. J Dent Educ. 2002; 66(9):102130 . 
22. Gorter R, Freeman R, Hammen S, Murtomaa H, Blinkhorn A, Humphris G. Psychological stress and health in undergraduate dental students: fifth year outcomes compared with first year baseline results from five European dental schools. Eur J Dent Educ. 2008; 12(2):61-8.

23. Musser LA, Lloyd C. The relationship of marital status and living arrangement to stress among dental students. J Dent Educ. 1985; 49(8):573-8.

24. Sugiura G, Shinada K, Kawaguchi Y. Psychological well-being and perceptions of stress amongst Japanese dental students. Eur J Dent Educ. 2005; 9(1):17-25.

25. Fonseca J, Divaris K, Villalba S, Pizarro S, Fernandez M, Codjambassis A, Polychronopoulou A. Perceived sources of stress amongst Chilean and Argentinean dental students. Eur J Dent Educ. 2013; 17(1):30-8.

26. Uraz A, Tocak YS, Yozgatligil C, Cetiner S, Bal B. Psychological well-being, health, and stress sources in Turkish dental students. J Dent Educ. 2013; 77(10):1345-55.

27. Divaris K, Mafla AC, Villa-Torres L, Sánchez-Molina M, Gallego-Gómez CL, Vélez-Jaramillo LF, Polychronopoulou A. Psychological distress and its correlates among dental students: a survey of 17 Colombian dental schools. BMC Med Educ. 2013; 13(1):91. Doi: 10.1186/1472-692013-91.

28. Goh AMY, Chiu E. Campus mental health: Are we doing enough? Asia Pac Psychiatr. 2009; 1(2):58-63.

29. Rajab LD. Perceived sources of stress among dental students at the University of Jordan. J Dent Educ. 2001; 65(3):232-41.

30. Yap AV, Bhole S, Teo CS. A cross-cultural comparison of perceived sources of stress in the dental school environment. J Dent Educ. 1996; 60(5):459-64.

31. Westerman GH, Grandy TG, Ocanto RA, Erskine CG. Perceived sources of stress in the dental school environment. J Dent Educ. 1993; 57(3):225-31.

32. Spielberger CD. The nature and measurement of anxiety. Em C. D Spielberger \& R. Diaz-Guerrero (Orgs.), Cross-cultural anxiety. 1976; 47-64.

33. Costa SDM, Durães SJA, Abreu MHNGD. Feminization of the odontology course at the State University of Montes Claros, Minas Gerais State. Cien Saude Colet. 2010; 15:1865-73.

34. Jayakody K, Gunadasa S, Hosker C. Exercise for anxiety disorders: systematic review. Br J Sports Med. 2014; 48(3):187196.

35. Gotink RA, Meijboom R, Vernooij MW, Smits M, Hunink MG. 8-week mindfulness based stress reduction induces brain changes similar to traditional long-term meditation practice - A systematic review. Brain Cogn. 2016; 108: 32-41.

36. de Witte M, Spruit A, van Hooren S, Moonen X, Stams GJ. Effects of music interventions on stress-related outcomes: a systematic review and two meta-analyses. Health Psychol Rev. 2019; 6: 1-62.

37. Dhama K, Gupta R, Singla A, Patthi B, Ali I, Niraj LK, Kumar JK, Prasad M. An insight into spiritual health and coping tactics among dental students; a gain or blight: a cross-sectional study. J Clin Diagn Res. 2017; 11: ZC33-ZC38.

\section{Correspondência para:}

Francisco Wilker Mustafa Gomes Muniz

e-mail: wilkermustafa@gmail.com

Rua Gonçalves Chaves, 457 Centro

96015-560 Pelotas/RS 